\title{
Sui confini della regione accessibile ai protoni riflessi dall'onda d'urto terrestre
}

(On the upstream wave boundary outside the Earth's bow shock)

\author{
I. Diodnto (*) \\ Ricevuto il 6 Gingno 1976
}

\begin{abstract}
Riassusto. - Si presenta un metodo che consente di deteminare la forma e l'estensione della regione in cui si propagano i protoni del vento solare riflessi dall'onda d'urto stazionaria della Terra. Si introduce un sistema di riferimento in eui la regione dei protoni riflessi ha una forma indipendente dai valori dei parametri che caratterizzano il vento solare: tale rappresentazione rende più agevole il confronto fra osservazioni eseguite in punti dello spazio e tempi differenti.
\end{abstract}

SunMary. - A method is deseribed for the determination of the shape and extension of the region containing solas wind protons reflected by the Earth's bow shock. A system of reference is introduced where the shape of the reflected proton region has a shape independent from the actual values of the solar wind parameters: such a representation makes easier the comparison among observations performed at different times and locations.

\section{INTRODUZIONE}

L'onda d'urto stazionaria, prodotta dalla interazione del vento solare con il campo magnetico terrestre, è stata oggetto di numerose investigazioni nel corso rlegli ultimi 15 ami. Una discussione delle sue proprieta, sia da un punto di vista osservazionale che teorico, è data in vari articoli di rassegna, fra cui ricordiamo Spreiter e Nlksne, $1969\left({ }^{26}\right)$, Wolfe e Intriligator, $1970\left(^{18}\right)$, Dobrowolny e Formisano, $1973\left(^{5}\right)$.

(*) Laboratorio per lo stulio del Plasma nello Spazio, C.X.R. FraseatiRoma. 
Un risultato notevole delle ricerche più recenti è quello di aver stabilito che alcune particelle del vento solare vengono "accelerate e riflesse " dall'onda d'urto ter'estre. Osservazioni di protoni "riflessi " con energia lino a $\sim 100 \mathrm{KeV}$, sono riportate cla $A$ sbrislge et al. ('), 1968 e Iin et al., 1974(13). Sembra anche accertato (Scarf et al., 1970)(14) che esista un legame fra queste particelle e le onde magnetoidrorlinamiche di lungo periodo osservate da vari esperimenti in una vasta regione dello spazio interplanetario "a monte " lell'onda d'urto (Greenstadt et al., 1968, Fairfield, 1969) (12,7).

Barnes, $1970\left(^{(3)}\right.$ e Fredricks, $1975\left(^{9}\right)$ hanno proposto meccanismi per la generazione di onde, con periodi dellordine di alcune decine di secondi, basati sulla interazione delle particelle del vento solare con i protoni riflessi dall'onda d'urto e in moto lungo le linee di forza del campo inagnetico interplanetario. Il processo di riflessione delle particelle da parte dell'onda d'urto è stato discusso da Somnerup $1969\left({ }^{15}\right)$ e Greenstault, 1975 (11).

Scopo del presente lavoro è di descrivere un metorlo che consente di determinare la forma e l'estensione della regione in cui ci si aspetta che si propaghino i protoni riflessi dallonda d'urto. Una prima applicazione di questo metodo ha fornito recentemente una stima della componente parallela al campo magnetico interplanetario della velocitì media dei protoni riflessi (1)iorlato et al., 1976) $\left({ }^{4}\right)$.

Ulteriori applicazioni sono discusse nel paragrafo 4 . In vista di esse sembra utile presentare qui brevemente le basi fisiche erl il formalismo matematico rel metorlo proposto.

\section{2. - DETERMINAZIONE DEI CONFINI DELIA REgIONE DEI PROTONI RIFI.ESSI}

Lo scopo del presente studio è quello di determinare i confini della regione ove si propagano i protoni riflessi dall'onda d'urto terrestre, sulla base di osservazioni di plasma e campo magnetico interplanetario effettinate ad un certo istante in un punto $P$ dello spazio.

Ise quantiti che occorre misurare sono:

$$
\begin{aligned}
& \overrightarrow{\mathrm{V}}=\text { velociti del vento solare; } \\
& \vec{N}=\text { densiti dei protoni del vento solare } \\
& \overrightarrow{\mathrm{B}}=\text { intensiti del campo magnetico interplanetario; } \\
& 0, \phi=\text { latitudine e longitudine solare-eclittica di } \vec{B} .
\end{aligned}
$$


I protoni riflessi si avvolgono intorno alle linee di forza del campo magnetico interplanetario. Poiché le linee di forza, congelate nel plasma, vengono trascinate da questo con la velocità $\vec{V}$ del vento solare, ci si aspetta che anche $\mathrm{i}$ protoni riflessi siano trascinati con la stessa velocità. Pertanto se indichiamo con $\vec{V}_{p}$ la velocità del centro di girazione dei protoni riflessi, possiamo serivere:

$$
\overrightarrow{\mathrm{V}}_{p}=\overrightarrow{\mathrm{V}}+p V \frac{\overrightarrow{\mathrm{B}}}{|B|}
$$

avendo in tal modo esplicitato la componente di $\vec{V}_{p}$ parallela al campo magnetico $\left(V_{\prime \prime}=p V\right)$. Fissati i valori di $V, N, B, \vartheta$, $\phi$, si trova che l'estensione della regione dei protoni riflessi e controllata dalla direzione di $\vec{V}_{p}$ e quindi clal solo parametro $p$, come è illustrato schematicamente dalla fig. 1 .

Eे conveniente nella discussione seguente riferirsi al piano $\pi$ passante per il punto $P$, parallelo alla direzione terra-sole (asse $X_{\mathrm{SE}}$ del sistema solare-eclittico) e al vettore $\vec{B}$. In questo piano consideriamo
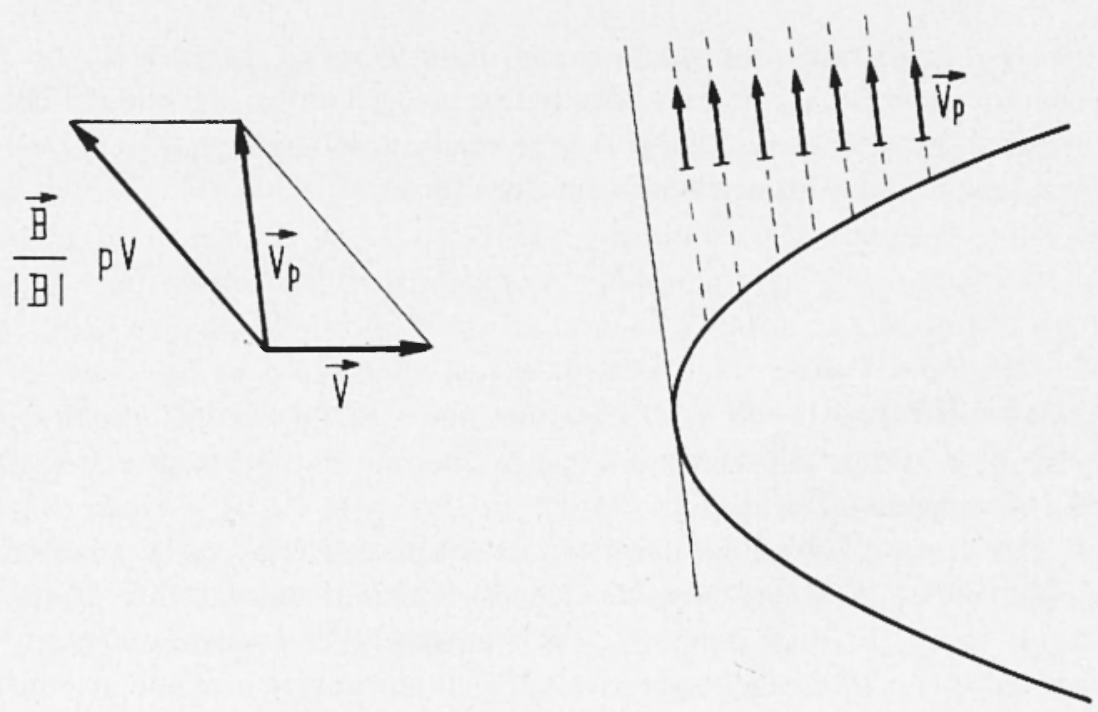

Fig. 1 - Il disegno mostra quale jegione dollo spazio è aceossibilo a protoni emergenti dallo shock con velocita del centro di gruila pari a $\vec{V}_{p}$. 
il sistema di coordinate $(x, \eta)$ introdotto da Greenstadt (1972) $\left({ }^{10}\right)$ per studiare la struttura dell'onda d'urto terrestre (l'asse $x$ d parallelo all'asse $X_{\text {SE }}$ e l'asse $\eta$ è ortogonale ad esso).

Isa determinazione della regione dei protoni riflessi viene effettuata nel morlo descritto qui di seguito:

\subsection{Caleolo della posizione dell'onda d'urto terrestre.}

I'onda d'urto puó esser rappresentata (cfr. per es. Jgoirli et al., $1970)\left(^{6}\right)$ da $u n$ paraboloide di rotazione avente la retta Terra-Sole come

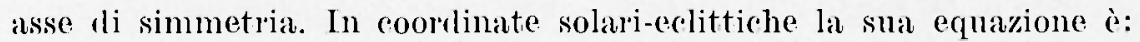

$$
Y^{2}+Z^{2}=U^{2}-X^{2}
$$

love $\varrho_{0}$ dic l'intersezione del paraboloide con l'asse $I_{S E}\left(0 \quad Z_{S E}\right)$ e $G_{r}=Q^{2} / D$ ove $I$ d̀ la distanza geocentrica del punto subsolare de] paraboloisle. L'intersezione de] paraboloide [2] con il piano $(x, \eta)$ è la parabola di equazione

$$
\eta^{2}=-G I+\left(Q_{0}^{2}-\left(q^{2}\right)\right.
$$

dove $q$ è la distanza del piano $(x, \eta)$ dalla Terra. I parametri o e $r$ vengono determinati in base alla teoria idrodinamica dellonda d'urto terrestre (Spreiter et al, 1968)(17) ed ai risultati delle osservazioni dirette compinte rai satelliti artificiali. Da Spreiter et al. si ha:

$$
D=\frac{C}{\left(N \cdot V^{2}\right)^{1 / 2}}\left(1+1,1 \frac{(\gamma-1) M^{2}+2}{(\gamma+1) M^{2}}\right)
$$

dove $\gamma$ i il rapporto dei calori specifici preso uguale a $5 / 3$, ed $M$ i il numero di Mack Alfvenico del vento solare: $M=\gamma /+\pi N m_{p} / B$ ( $m_{p}=$ massa del protone).

Ia costante $Q$ iene determinata empiricamente dalla posizione media dell'onda d'urto terrestre: le osservazioni del satellite Heos-1 (Eggidi et al., 1970) (6) danno $G=95$ se nella [4] $N$ è misurata in ( $^{\circ}{ }^{3}$, $Y$ in $\mathrm{km} / \mathrm{sec}$ e $D$ in raggi terrestri. Anche il parametro $g_{0}$ viene ot tenuto empiricamente, in quanto le osservazioni dirette indicano che, al variare della pressione dinamiea del vento solare $X r^{2}$, il rapporto $o_{0} / D$ si mantiene approssimativamente costante $(=1,67)$. 
2.2. Calcolo della equazione della retta che limita la regione dei protoni riflessi.

Nella [1] si può assumere che $\vec{V}$ sia antiparallela all'asse $X$, dal momento che la componente azimutale della velocità del vento solare è in prima approssimazione trascurabile rispetto a quella radiale.

La regione del piano $\pi$ che può essere raggiunta dai protoni riflessi è quella compresa fra la parabola [3] ed una semiretta tangente alla parabola e parallela alla direzione di $\vec{V}_{p}$ (fig. 1). Un semplice calcolo mostra che l'equazione della tangente $t$ cercata $\grave{e}$

$$
\eta=a \chi+\beta
$$

dove ̀̀

$$
\alpha=\frac{P B \eta}{P B_{X}-B}
$$

e

$$
\hat{\rho}=\frac{4 \alpha^{-} q^{-}-4 \alpha^{-} Q^{2}{ }_{0}-G^{-2}}{4 \alpha G^{2}}
$$

\section{NormalizZaZione Della Regione Del protoNi riflessi}

Le equazioni [3] e [5] permettono di calcolare la regione dove si propagano i protoni riflessi dall'onda d'urto, in funzione del parametro $p$. Tutavia, quando si voglia confrontare fra loro osservazioni eseguite in condizioni fisiche differenti (ciò̀ con valori diversi delle quantità $r$, $N, B, \vartheta$ e $\phi$ ) insorge una difficoltà, legata al fatto che sia la posizione dell'onda d'urto (equazione 3 ) che quella della retta $t$ (equazione 5) dipendono dai valori delle suddette quantità. Il confronto risulta più agevole, soprattutto da un punto di vista grafico, se si proietta la fig. 1. su un piano $\pi^{\prime}$ in cui la regione di protoni riflessi abbia una forma prefissata, per un dato valore del parametro $\%$. Introduciamo, perciò, in tal piano un riferimento $\left(x^{\prime}, \eta^{\prime}\right)$ in cui la proiegione della parabola [3] sia rappresentata dalla equazione

$$
\eta^{\prime 2}=-a x^{\prime}
$$

Il passaggrio dal piano $(x, y)$ al piano $\left(x^{\prime}, \eta^{\prime}\right)$ si realizza, allora, con una trasformazione del tipo: 


$$
\begin{gathered}
x^{\prime}=\frac{\lambda^{2} G}{a} x+\frac{\lambda^{2}}{a}\left(q^{2}-\varrho^{2}{ }_{0}\right) \\
\eta^{\prime}=\lambda \eta
\end{gathered}
$$

dove $\lambda$ è un parametro il cui valore risulta determinato dalla ulteriore condizione che la retta $t^{\prime}$, proiezione della retta $t$, abbia un coefflciente angolare prefissato $m^{\prime}$.

Nel riferimento $\left(x^{\prime}, \eta^{\prime}\right)$ la retta $t^{\prime}$ avrà equazione

$$
\eta^{\prime}=\frac{\alpha \cdot a}{\lambda \cdot G} x^{\prime}+\frac{\lambda \alpha}{G}\left(Q_{0}^{\circ}-q^{2}\right)+\beta
$$

rla cui:

$$
\lambda=\frac{a \cdot a}{m^{\prime} G}-\frac{a}{m^{\prime} G} \cdot \frac{p B \eta}{p B_{X}-B}
$$

le equazioni [9] e [11] definiscono il riferimento cercato $\left(x^{\prime}, \eta^{\prime}\right)$, in cui la regione dei protoni riflessi è "normalizzata " arl una forma prefissata, dipendente solo dal parametro $p$. Il contorno di questa regione ì definito dalla parabola di equazione [9] e dalla retta di equazione [10].

E da sottolineare che la trasformazione [9] non muta il carattere ri appartenenza o meno alla regione dei protoni riflessi.

La fig. 2a (2b) tratta da Diodato et al., $1976\left(^{(4)}\right.$ mostra le proiezioni sul piano $\left(x^{\prime}, \eta^{\prime}\right)$ (lelle posizioni in cui Heos-1 (non ha) osservato " $u p$ streum waves" che si ritengono con molta probabilità legate all'esistenza di protoni riflessi.

\section{4. - Conclusioni}

Il metorlo descritto sopra permette di determinare la regione rlei protoni riflessi dall'onda d'urto terrestre, qualora siano noti i parametri del vento solare (densità e velocità del plasma, intensità e direzione del campo magnetico) in un punto dello spazio interplanetario. Inoltre, la trasformazione [9] definisce un sistema di riferimento in cui la regione dei protoni riflessi ha una forma fissata (dipendente soltanto dal paramentro $p$ ), il che rencle più agevole il confronto fra le osservazioni eseguite in posizioni e istanti diversi.

Il metorlo si rivela utile soprattutto per effettuare sturli statistici relle proprietà del plasma nella regione dei protoni riflessi (questa 
regione viene spesso chiamata "upstream wave region", dato che, come menzionato nella introduzione, i protoni riflessi sembrano essere associati ad onde magnetoidrodinamiche di bassa frequenza).

Diodato et al., 1976 hanno applicato il presente metodo per valutare il parametro $p$. Allo scopo sono stati impiegati i dati di plasma e campo magnetico forniti dal satellite europeo Heos-1 e si è fatta l'ipotesi che la presenza (o assenza) delle "upstream uaves" fosse indice della presenza (o assenza) dei protoni riflessi dall'onda d'urto.

Rappresentando le osservazioni nel piano $\left(x, \eta^{\prime}\right)$, ̀̀ risultato che la retta $t^{\prime}$ corrispondente a $p=2$ minimizzava il numero di "error $i$ " (intendendosi per "errore" una osservazione di onde nella regione "proibita" ai protoni riflessi ovvero l'assenza di onde nella regione dei protoni riflessi). Una analisi più dettagliata ha anche suggerito che $p$ vari al variare del punto di riflessione lungo la superficie dell'onda d'urto da $\sim 1.6$, intorno al punto subsolare, fino a $\sim 2.6$ sui fianchi.

Fra le possibili ulteriori applicazioni, segnaliamo:

a) Interazione del vento solare con i protoni riflessi. Aleuni autori (Amata e Formisano, 1976, Auer et al., 1976) ${ }^{8,2}$ ) hanno suggerito che le proprietà del vento solare possano essere modificate in modo significativo dalla presenza dei protoni riflessi. Fino ad oggi, tuttavia, manca uno studio sistematico del fenomeno. Tale studio può esser compinto, disponendo di dati di plasma e campo magnetico registrati simultaneamente da due satelliti attraverso correlazioni fra i valori (lei parametri del vento solare osservati nella regione interplanetaria "imperturbata" e in quella dei protoni riflessi. Questa può esser determinata con le equazioni [3] e [5], usando per $p$ il valore medio 2 calcolato da Diodato et al., $1976\left(^{4}\right)$.

b) Mecanismi di generazione delle "upstream waves". Barnes $(1970)\left({ }^{3}\right)$ e Friedricks $(1975)\left({ }^{9}\right)$ hamo proposto teorie differenti per la produzione di onde magnetoidrodinamiche dalla interazione del vento solare con i protoni riflessi. Osservazioni dettagliate di plasma e campo magnetico possono permettere di verificare quale dei meceanismi ̀̀ effettivamente operante. Di nuovo, le equazioni [3] e [5] permettono di stabilire a quale regione si riferiscono le osservazioni.

Concludendo, si può sottolineare l'importanza di un approfoncimento della nostra conoscenza (attualmente molto limitata) delle caratteristiche della regione dei protoni riflessi dall'onda d'urto terrestre, regione che ha un'estensione perlomeno confrontabile con quella 


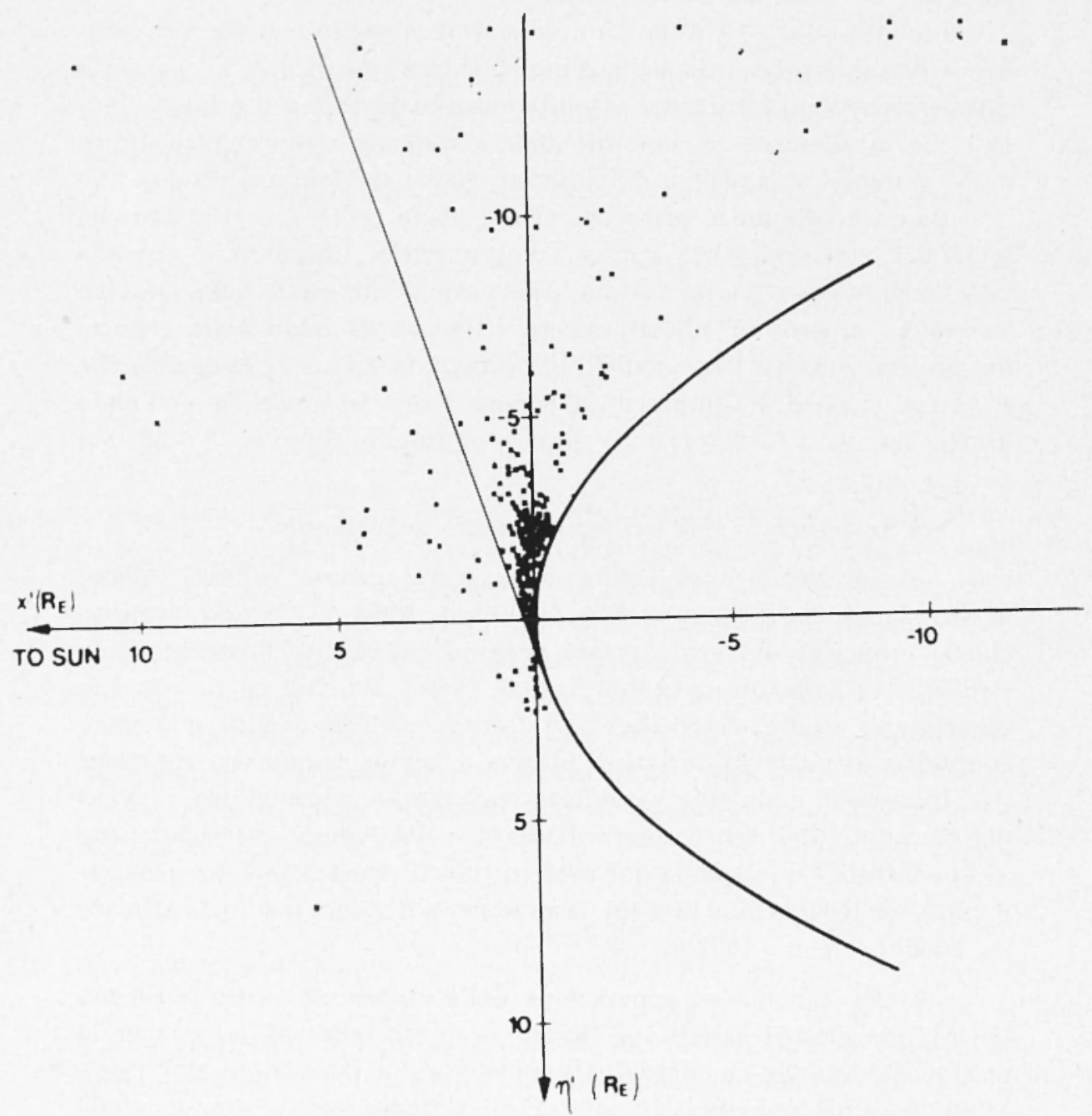

Fig. 2a

Fig. 2(a,b) - Posizione del satellite nol sistema $\left(x^{\prime}, \eta^{\prime}\right)$ (vedi testo) in cui sono state osservate (fig. 2a) o non sono state osservite (figr. 2h) "upstream waves". Ial parabola rappresenta la sexione dell'ondid di urto terrest re deseritta dalla [8]. La tangente alla parabola delimita la regione ove le "upstream waves" dovrebhero essere osservate per $p-2$, (da Diodato et al. 1976)( $\left.{ }^{4}\right)$. 


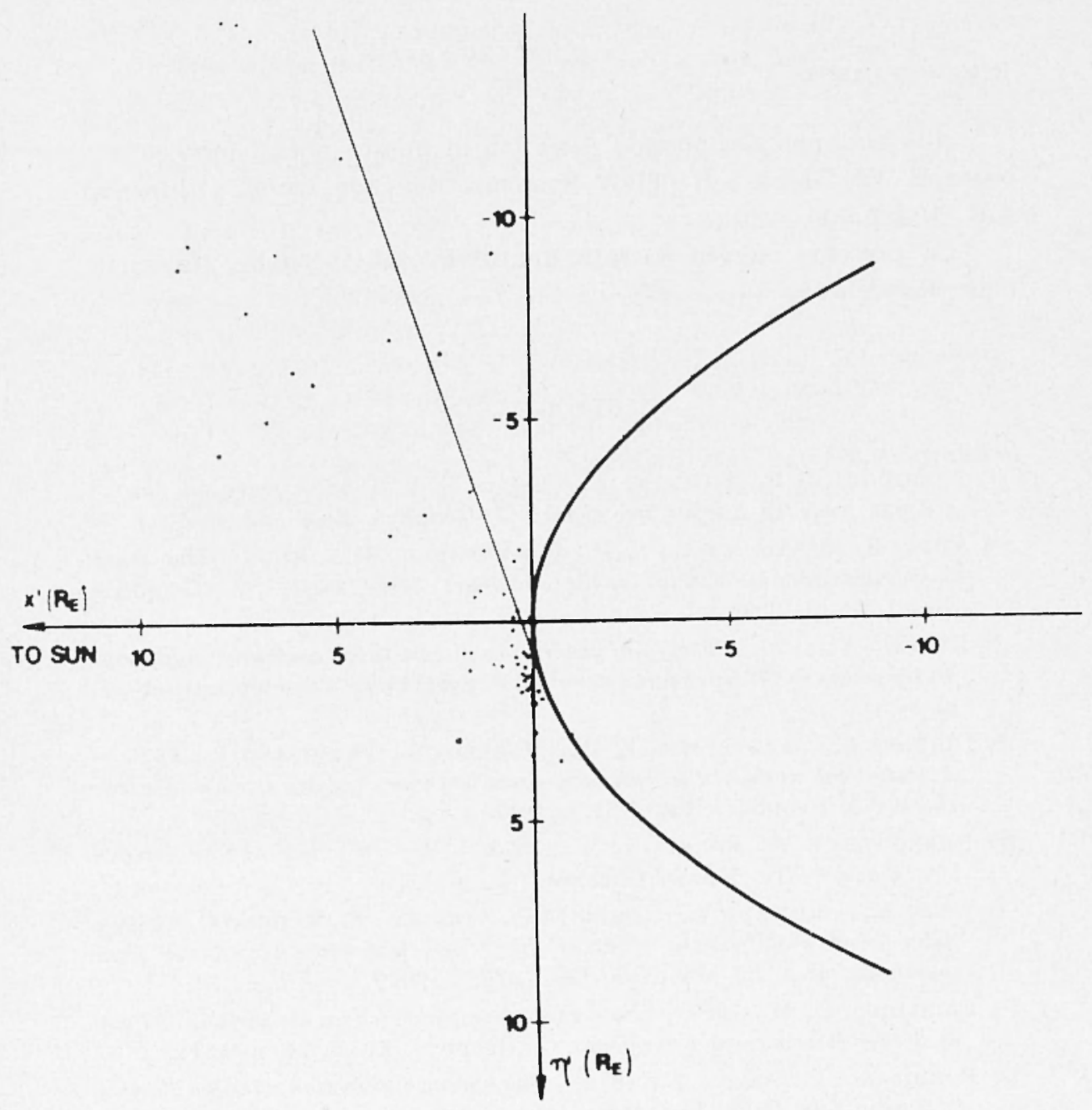

Fig. 2b - Veri didascalia sotto fig. 2a. 
della magnetosfera. Il metorlo clescritto in questa nota può costituire un utile strumento per lo sviluppo di tali ricerche.

\section{RINGRAZIAMENTI}

Allo sviluppo del metodo descritto in questa nota hamno contribuito E. W. Greenstarlt (TRW Systems, Redondo Beach, California) e G. Moreno.

La presente ricerca è stata finanziata dal Consiglio Nazionale delle Ricerche.

\section{BIBIJOGRAFIA}

(1) Asbringe, J. R., Baye, S. J., Stroxg I. B., 1968. - Oulward flow of protons from the Earlh's bou shock, "J. Geophys. Res.", 73, p. 5777.

(2) Auer, R. I)., Grüwat,d, H., Rosexpauer, HI., 1976. - Bow shock associated proton heating in the upstream Solar wind, "I. Geophys. Res.", 81, p. 2030.

(3) BARNes, A., 1970. - Theory of generation of bow-shock associated hydromagnetic uaves in the upstream interplanetary medium. "Cosmic Electrol", 1 , p. 90.

(4) Diodato L.,, Greexstant, E. W., Moreno, G., Formisano V., 1976. A statistical study of the upstream wave boundary outside the Eartlis bow shock, "J. Geophys. Res.", 81, p. 199.

(5) Dobrowotxy M., Formisano V., 1973. - The structure of the Earth's bow shock. "Riv. Nuovo Cimento", 3, p. 419.

(6) Egidi A., Formisano V., Pal.miotto P., Saracexo l'., Morexo G., 1970. Solar wind and location of shock front and magnetopause at the 1969 solar maximum, "J. Geophys. Res.", 75, p. 6999.

(7) FaIRFIELD, D. M., 1969. - Bow shock associated waves observed in the far upstream interplanetary medium. "J. Geophyss. Res.", 74, p. 3541.

(8) Formisaso V., AMata F., 1976. - Solar wind interaction with the Eartlis Magnetic field 4. Preshock Perturbation of the Solar Wind. "J. Geophys. Ros.", in press.

$\left({ }^{9}\right)$ FRIEDRICKs, R. W..1 975. - A model for generation of bow-shock associaled upstream waves. "J. Geophys. Res", 80, p. 7 .

(10) Gressstadt, E. W., 1972. - Binary inder for assessing local bow shock obliquily. "J. Geophys. Res.", 77, p. 5467.

(11) GRExs'TADT, E. W., 1975. - The upstream escape of energized solar wind prolons from the bow shock. "The IIagnetospheres of the Earth and Jupiter", p. 3 ed. by V. Formisano, Reidel-Dordrecht. Holland. 
(12) Greenstant, E. W., Greex, I. M., Inoure, G. T., Huxdmalsex, A. J., BAME, S.J., STrosi, I. B., 1968. - Correlated magnetic field and plasma observations of the Earth's bow shock. "J. Geophys. Res.", 73, p. 51.

(13) Iin, R. P., Mrwa, C., Anderson, K. A., 1974. - 30 to 100 KeV protons upstream from the Earth's bow shock. "J. Geophys. Res.", 79, p. 489.

(14) Scarf, F. I., Friedricks, R. W., Frask, I. A., Russell, C. T., Colemax, P.J., Neugenauer, M., 1970. - Direct correlations of large-amplitude uaves, with suprathermal protons in the upstream solar wind. "J. Geophys. Res.", 75, p. 7316.

(15) SONNERUP, B. U. O., 1969. - Acceleration of particles refected at a shock front. "J. Geophys. Res.", 74, p. 1301.

(16) Spreited, I. R., Alkswe, A. Y., 1969. - Plasma flow around the magneto. sphere. "Rev. Geophys.", 7, p. 11.

(17) Spreiter, J. R., Alksxe, A. Y., Summers, A. L., 1968. - E.rternal aerodynamics of the magnetosphere. "Physics of the Magnelosphere", p. 301 ed. by. R. I. Carovillano, Reidel-Dordrecht. Holland.

(18) Wolfe, J. H., Ixtrif.igator, D. S., 1970. - The solar wiml interaction with the geomagnetic field. "Space Sci. Rev.", 10. 1. 5ll. 